\title{
Replacement of Maternal 40S rRNA Precursor by Newly Synthesized Precursor in Early Embryos of Xenopus laevis
}

\author{
Nobuaki Furuno*, Koichiro Shiokawa and Hideki Kobayashi \\ Department of Biology, Faculty of Science, Kyushu University 33, Fukuoka \\ 812, Japan
}

\begin{abstract}
The amount of intact 40S rRNA precursor was followed by Northern hybridization in the course of the early embryogenesis of wild-type Xenopus laevis and its anucleolate mutant.

The total amount of 40S rRNA precursor did not alter appreciably until the midblastula stage, decreased at the late blastula stage, and then increased. In the anucleolate mutant, in which no rRNA synthesis occurs, the 40S rRNA precursor decreased at the late blastula stage and disappeared after the gastrula stage. In the nuclear fraction of the wild type, the 40S rRNA precursor was detectable after the midblastula stage.

Therefore, the 40S rRNA precursor in the pre-blastula embryos is maternal and decreases at the late blastula stage. New synthesis of 40S rRNA precursor apparently occurs after the midblastula stage.
\end{abstract}

During Xenopus embryogenesis, the synthesis of rRNA is uniquely regulated (5). In cleaving embryos, rRNA synthesis is suppressed, but after the midblastula transition, rRNA is actively synthesized $(8,13)$. Although there is evidence for mature rRNAs (28S, 18S, and 5.8S) during Xenopus embryogenesis, the 40S rRNA precursor, which is the primary transcript of rRNA genes (6), has not been well characterized during Xenopus embryogenesis. Investigation of this precursor during embryogenesis is necessary to better comprehend the regulation of rRNA synthesis during development. Since the 40S rRNA precursor is rapidly processed (4), detection of this RNA has been regarded as a signal of rRNA synthetic activity during embryogenesis.

However, at the pre-blastula stage, when rRNA synthesis is considered to be suppressed, the $5^{\prime}$ end of 40S rRNA precursor was evidenced by S1 protection in the cytoplasm (3), a finding which suggested the existence of a maternal 40S rRNA precursor in pre-blastula embryos. However, it is not clear whether the intact 40S rRNA precursor persists during embryogenesis, nor whether these molecules are from the oocyte or are newly synthesized.

To differentiate between the maternal 40S rRNA precursor and the newly synthesized one, the use of an anucleolate mutant which lacks the rRNA synthetic ability in embryogenesis (2) should be ideal. Using this mutant, we investigated the 40S

\footnotetext{
* Present address: Department of Molecular Biology, Graduate School of Medical Science, Kyushu University, Fukuoka 812, Japan
} 
rRNA precursor during Xenopus embryogenesis.

In this report, we describe the replacement of maternal 40S rRNA precursor by newly synthesized precursor during early embryogenesis in $X$. laevis. We found that the 40S rRNA precursor detected before the blastula stage is maternal and is retained without processing or degradation until the blastula stage. After the blastula stage, there is a gradual decrease and it is not detected at the gastrula stage, which suggests that the 40S rRNA precursor present after the midblastula stage is newly synthesized. This was confirmed by the evidence that 40S rRNA precursor in the nucleus, not detected in early embryos, is first observed after the midblastula stage.

\section{MATERIALS AND METHODS}

Embryos. Fertilized eggs obtained by injecting Xenopus laevis with chorionic gonadotropin were dejellied, and their embryonic stages were synchronized by collecting only 2-celled embryos of the same batch. These embryos were allowed to develop at $21^{\circ} \mathrm{C}$ to the required stages (9). Embryos homozygous for anucleolate mutation were obtained from a pair of heterozygous male and female. One-fourth of the sibling embryos from a cross between heterozygotes $(+/ 0 \mathrm{nu} ; 1-\mathrm{nu})$ are expected to be anucleolate mutants $(0 / 0 \mathrm{nu} ; 0-\mathrm{nu})$. At the tailbud stage, a small portion of the tailbud was removed from each embryo for identification of the nucleolus genotype by phase contrast microscopy.

Preparation of the nuclear fraction. The nuclear fraction of embryonic cells was obtained by Woodland's method (17) with slight modification. Embryos were rinsed once with $20 \mathrm{mM}$ Tris- $\mathrm{HCl}$ (pH 7.4), $0.5 \mathrm{M} \mathrm{KCl}, 4 \mu \mathrm{g} / \mathrm{ml}$ polyvinylsulfate (PVS), and $0.5 \%$ Triton $\mathrm{X}$ 100 , and then homogenized in the same buffer using a Dounce B homogenizer. Pellet and soluble fractions were obtained by centrifuging the homogenate at $3,000 \mathrm{rpm}$ for $5 \mathrm{~min}$. This pellet served as the nuclear fraction.

RNA extraction. Embryos were frozen with liquid nitrogen and disrupted with a glass rod in $0.1 \mathrm{M}$ sodium acetate ( $\mathrm{pH}$ 5.0) containing $0.5 \%$ sodium dodecyl sulfate (SDS) and $5 \mu \mathrm{g} / \mathrm{ml}$ PVS. Embryonic RNAs were extracted as described (12), precipitated in $70 \%$ ethanol, and stored in 70\% ethanol until use. RNAs recovered from embryos were almost the same in quantity, irrespective of the stage. RNAs were also extracted from the nuclear and soluble cytoplasmic fraction, using the same procedure.

Northern hybridization. Two recombinant plasmids, pXlr14D which contains the region complementary to the $5^{\prime}$ end specific to the 40S rRNA precursor (1) and pXIrT31 which contains the external transcribed spacer and a portion of the 18S rRNA gene, were used as probes for Northern hybridization (Fig. 1). pXIrT31 was subcloned from pXIr101A (1) in our laboratory. These plasmids were nick-translated with $\left[\alpha-{ }^{32} \mathrm{P}\right] \mathrm{dCTP}(10)$ and used as probes.

Northern hybridization was performed according to Thomas (15) with slight modification. RNAs were denatured in glyoxal, subjected to electrophoresis in $1.1 \%$ agarose gel in $10 \mathrm{mM}$ sodium phosphate buffer ( $\mathrm{pH}$ 7.0), and then transferred to a nylon filter (Biodyne ${ }^{\mathrm{TM}}$, BNNG, $1.2 \mu \mathrm{m}$, Japan Pall Co. Ltd.).

The filters were baked for $1.5 \mathrm{~h}$ at $80^{\circ} \mathrm{C}$ and treated with $20 \mathrm{mM}$ Tris- $\mathrm{HCl}(\mathrm{pH} \mathrm{8.0)}$ at $100^{\circ} \mathrm{C}$ for $5 \mathrm{~min}(16)$ to remove the glyoxal. RNAs on the filters were then hybridized with the probes, ${ }^{32} \mathrm{P}-\mathrm{pX} \operatorname{lrT} 31$ or $\mathrm{pX} \operatorname{lr} 14 \mathrm{D}$, in $50 \%$ formamide, $0.1 \%(\mathrm{w} / \mathrm{v})$ each of bovine serum albumin, polyvinylpyrrolidone and Ficoll-400, $0.3 \%$ SDS, $0.9 \mathrm{M} \mathrm{NaCl}, 50 \mathrm{mM}$ sodium phosphate (pH 8.3), $5 \mathrm{mM}$ EDTA, and $200 \mu \mathrm{g} / \mathrm{ml}$ E. coli tRNA for $18 \mathrm{~h}$ at $42^{\circ} \mathrm{C}$. Filters were washed three times with $2 \mathrm{xSSC}$ and $0.1 \%$ SDS at room temperature and then with $0.1 \times \mathrm{SSC}$ and $0.1 \% \mathrm{SDS}$ at $50^{\circ} \mathrm{C}$ for $30 \mathrm{~min}$. For autoradiography, the filters were air-dried and exposed to Fuji X-ray film (Fuji RX-50). 


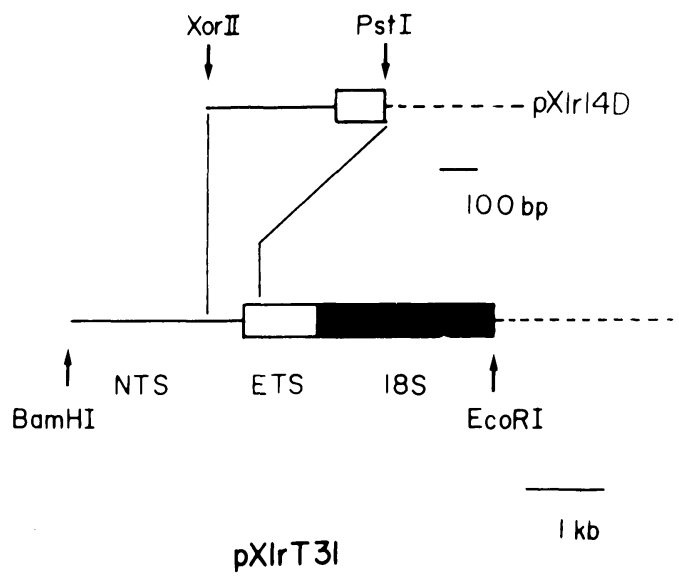

Fig. 1. Structure of two recombinant plasmids containing Xenopus laevis rRNA gene. bp, base pair. $\mathrm{kb}$, kilo base pair. NTS, nontranscribed spacer. ETS, external transcribed spacer. 18S, a part of the region coding for 18S rRNA. pXlrT31 carries $3.4 \mathrm{~kb}$ of a BamHI-EcoRI fragment which contains ETS, a part of the NTS, and a part of the 18S rRNA gene. The fragment was inserted in the BamHI-EcoRI site of pBR322. pXlr14D carries a 433-bp fragment bounded by a PstI site at -320 and an XorII site at +113 $\mathrm{bp}$; it was inserted in the PstI-XorII site of pBR322.

\section{RESULTS}

Detection of the 40S $r R N A$ precursor in Xenopus wild-type embryo. To identify the 40S rRNA precursor, RNAs were extracted from neurulae, at which stage rRNA is actively synthesized, and hybridized with nick-translated ${ }^{32} \mathrm{P}-\mathrm{pX}$ XIT 31 DNA. As shown in Fig. 2, lane 1, three radioactive bands were obtained. The band indicated by the arrow was identified as a 40S rRNA precursor for the following reasons: (1) this band was not observed in the 0-nu mutant, which did not synthesize rRNA (Fig. 2, lane 2); (2) this band was observed with ${ }^{32} \mathrm{P}$-labeled pXlr14D, which contains only the external transcribed spacer specific for the 40S rRNA precursor (Fig. 2, lane 3); and (3) this band was not detected after treatment with sodium hydroxide (Fig. 2, lane 4). The lower molecular weight band corresponds to $18 \mathrm{~S}$ rRNA (Fig. 2, lane 2) and the high molecular weight band corresponds to the genomic DNA (Fig. 2, lane 4), since the RNA fraction of lane 1 was treated with sodium hydroxide for Southern hybridization (14).

RNAs were extracted from unfertilized eggs or embryos at various stages and hybridized with ${ }^{32} \mathrm{P}$-pXIrT31 DNA as a probe. As shown in Fig. 3, lanes 1-4, the 40S rRNA precursor was detected throughout the cleavage stages, during which rRNA synthesis is suppressed. Judging from the relative intensity of the band, the amount of $40 \mathrm{~S}$ rRNA precursor does not seem to change significantly during the period between fertilization and stage 8 (midblastula stage). During the blastula stages (Fig. 3, lanes 4 and 5), the intensity of the 40S rRNA precursor band decreased to about $30 \%$ at stage 8 and then increased rapidly at and after stage 9 (Fig. 3, lanes 5-7). At the gastrula stage (stage 10), the amount of 40S rRNA precursor is at the same level at that in cleaving embryos, and a large amount of 40S rRNA precursor was observed in the neurulae. Three independent experiments were carried out 


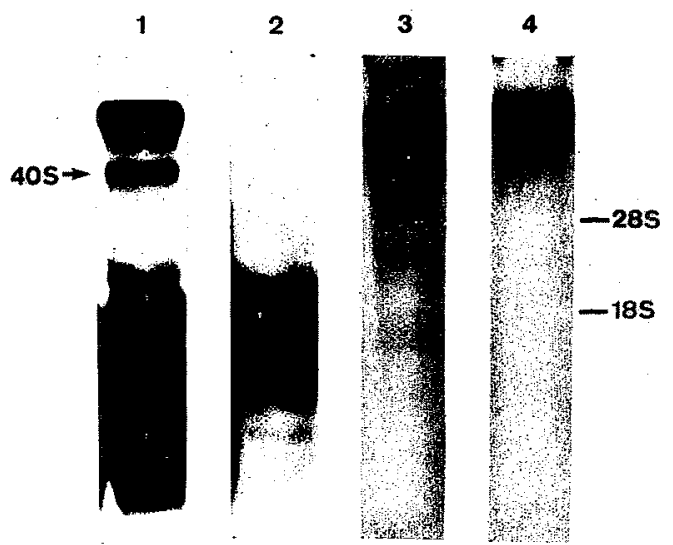

Fig. 2. Identification of the 40S rRNA precursor. Lane 1; RNAs extracted from neurulae (5 embryos) were hybridized with ${ }^{32} \mathrm{P}$-pXlrT31 DNA $\left(7 \times 10^{8} \mathrm{cpm} / \mu \mathrm{g}\right)$. Lane 2; RNAs from 0-nu ( 5 embryos) were hybridized with ${ }^{32} \mathrm{P}$-pXIrT31 DNA. Lane 3; RNAs from tailbud (5 embryos) were hybridized with ${ }^{32} \mathrm{P}$-pXlr 14D DNA $\left(2 \times 10^{8} \mathrm{cpm} / \mu \mathrm{g}\right)$. Lane 4 ; neurula DNAs ( 5 embryos) free from contaminating RNAs were hybridized with ${ }^{32} \mathrm{P}-\mathrm{pXI}$ 14D DNA according to Southern hybridization. The positions of $28 \mathrm{~S}$ and $18 \mathrm{~S}$ rRNAs are shown on the right.

and the results were reproducible.

Disappearance of maternally inherited $40 S \mathrm{rRNA}$ precursor in the anucleolate embryo. Since an anucleolate mutant (0-nu), which can develop into a tadpole,

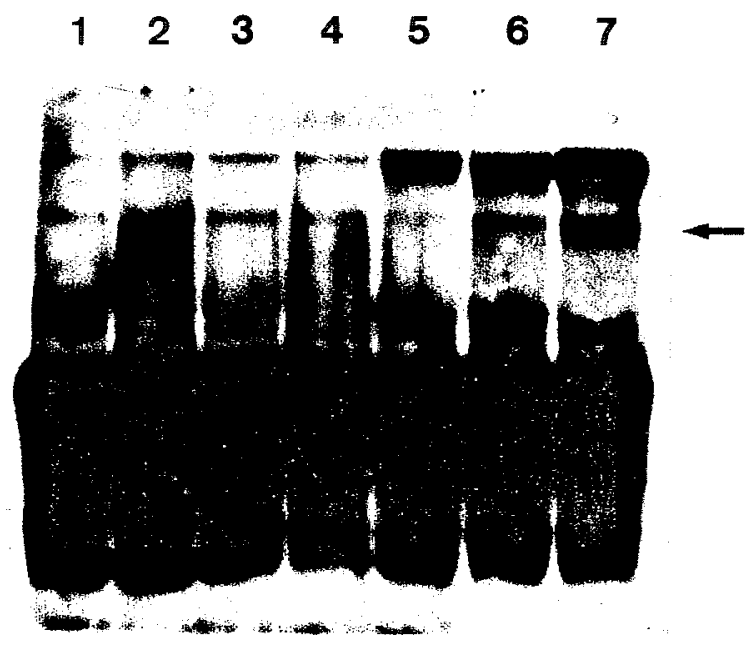

Fig. 3. Autoradiograph of blotted RNA extracted from unfertilized eggs or embryos at various stages. The RNAs extracted from five eggs or embryos were electrophoresed, transferred to a nylon filter, and hybridized with ${ }^{32} \mathrm{P}$-pXIrT31 DNA $\left(7 \times 10^{8} \mathrm{cpm} / \mu \mathrm{g}\right)$. The filter was exposed for one day. Lane 1; unfertilized egg. Lane 2; cleavage (stage 2-3). Lane 3; cleavage (stage $61 / 2$ ). Lane 4; midblastula (stage 8). Lane 5; late blastula (stage 9). Lane 6; gastrula stage (stage 10 1/2). Lane 7; neurula stage (stage 16). The arrow shows the position of the 40S rRNA precursor. The band which migrated slower than $40 \mathrm{~S}$ rRNA is the acridine orange-stainable DNA band. 


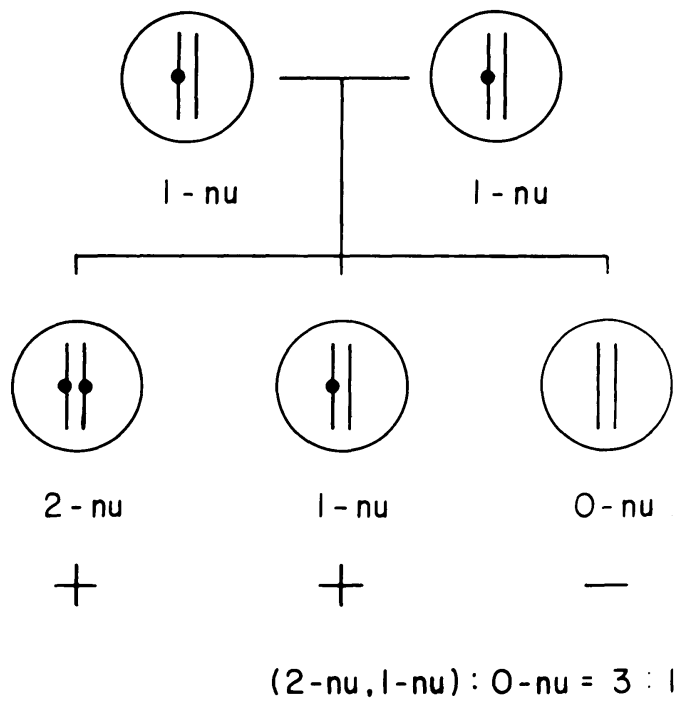

Fig. 4. Schematic diagram of events with a mating heterozygotic male and female. When a cross between heterozygotes (1-nu $\times 1$-nu) was made, three genotypes of embryos were obtained (25\% 2-nu, 50\% 1-nu, 25\% 0-nu). Although 0-nu embryos were not distinguishable phenotypically by the nucleolar numbers in early emrbryogenesis before neurula, one-fourth of the total progeny was expected to be 0-nu embryo. rRNA synthes is indicated by + , no synthesis by - . The closed circle represents a nucleolus.

does not synthesize rRNA during embryogenesis (2), the 40S rRNA precursor in 0 -nu embryos, if present, would be maternal.

To determine the origin and fate of the 40S rRNA precursor in the cleaving embryo, the 40S rRNA precursor in the 0 -nu embryo was investigated. We analyzed embryos obtained from heterozygotic mating (1-nu $\times 1-\mathrm{nu})$, since the genotype cannot be identified at the cleaving stage. Half of the embryos obtained by mating heterozygotic males and females should be heterozygotes (1-nu), and the other half should be homozygotes ( 0 -nu and 2-nu). Thus, one-fourth are expected to be 0 -nu embryos, which fail to synthesize rRNA (Fig. 4).

TABLE 1. PRESENCE OF 40S rRNA PRECURSOR IN SIBLING EMBRYOS FROM THE HETEROZYGOTE.

\begin{tabular}{lcc}
\hline \multirow{2}{*}{ Stage } & \multicolumn{2}{c}{ Number of embryos } \\
\cline { 2 - 3 } & Detected & Not detected (a) \\
Cleavage & 15 & 0 \\
Late blastula & $20(\mathrm{~b})$ & 0 \\
Gastrula & 16 & 4 \\
Neurula & 16 & 4 \\
Tadpole & 15 & 5
\end{tabular}

(a): The 40S rRNA precursor was not present on the autoradiogram.

(b): The 40S rRNA precursor was detected in all embryos, but the amount of the 40S rRNA precursor of five embryos was much less.

Twenty sibling embryos were analyzed individually at each stage, except that only fifteen were analyzed at the cleavage stage. The experiment was carried out as described in Materials and Methods. 
About twenty sibling embryos obtained by mating between male and female heterozygotes were analyzed individually at various stages (Fig. 5, A-E). The bands of 40S rRNA precursor can be observed in all five cleaving embryos (Fig. 5A). The intensity of these bands was much the same. However, at the late blastula stage, the density of the radioactive $40 \mathrm{~S}$ rRNA precursor band of two embryos (Fig. 5B, lanes 4 and 5 ) is very faint. The $40 \mathrm{~S}$ rRNA precursor band was not observed in some embryos analyzed at the gastrula stage (Fig. 5C, lane 4), neurula stage (Fig. 5D, lanes 1, 2 and 4), and tadpole stage (Fig. 5E, lane 3). These results are summarized in Table 1 . All the embryos have the 40S rRNA precursor at cleavage and late blastula stage, whereas at gastrula, neurula, and tadpole stages, about one-fourth of the embryos have no 40S rRNA precursor. The amount of 40S rRNA precursor is less than that of the other one in the late blastula stage embryos. These embryos can be regarded as 0 -nu mutants.

These results show that the $40 \mathrm{~S}$ rRNA precursor in cleaving stage embryos is maternal, decreases at the late blastula stage, and it is no longer detectable at the gastrula stage.

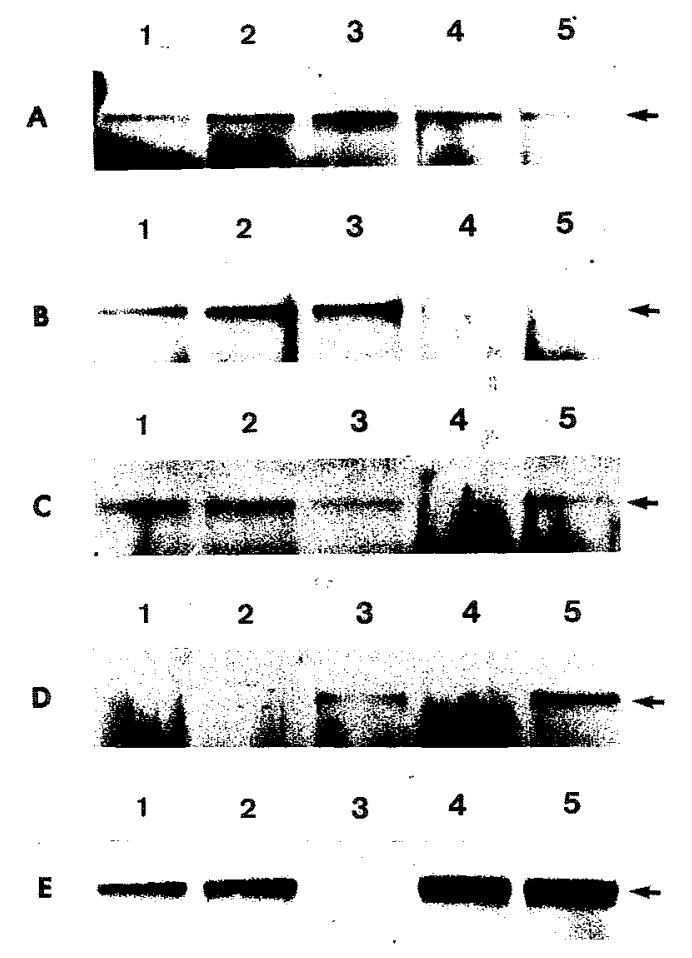

Fig. 5. The fate of 40S rRNA precursor in various stages of embryos derived from a cross between heterozygotes (1-nu $\times 1-n u)$. Embryos were obtained from the progeny of a cross between heterozygotes (see Fig. 4). RNAs were extracted individually from each embryo, electrophoresed, transferred to a nylon filter, and hybridized with ${ }^{32} \mathrm{P}-\mathrm{pXIrT} 31$ DNA $\left(1 \times 10^{8} \mathrm{cpm} / \mu \mathrm{g}\right)$. At each stage, $15-20$ embryos were analyzed, but only a part ( 5 embryos at each stage) is shown. Arrows indicate the 40S rRNA precursor. A, cleaving embryos (stage 6). B, late blastulae (stage 9). C, mid-gastrulae (stage 12). D, mid-neurulae (stage 18). E, tadpoles (stage 42). The filters of A, B, C, D, and E were exposed for 7 days, 5 days, 10 days, 1 day, and 1 day, respectively. 


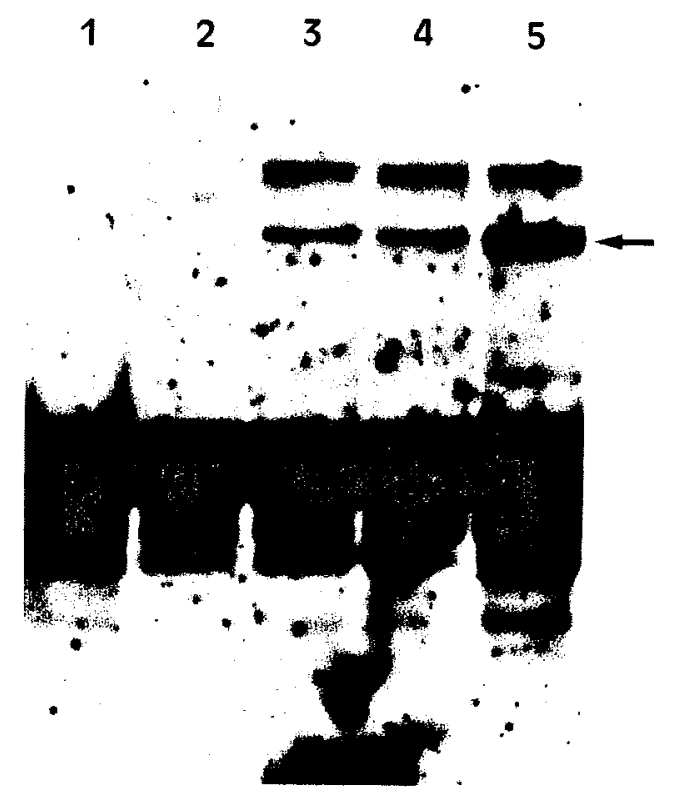

Fig. 6. Analysis of nuclear $40 \mathrm{~S}$ rRNA precursor during embryogenesis. Embryos at various stages were fractionated, as described in MATERIALs and METHODS. RNAs in the nuclear fraction were hybridized with ${ }^{32} \mathrm{P}-\mathrm{pXIrT31}\left(6 \times 10^{8} \mathrm{cpm} / \mu \mathrm{g}\right)$ DNA. The filter was exposed for five days. The arrow indicates the 40S rRNA precursor. Lane 1; cleavage (stage 3). Lane 2; midblastula (stage 8). Lane 3; late blastula (stage 9). Lane 4; gastrula (stage 10). Lane 5; neurula (stage 18). Recovery of DNAs from the nuclear fraction was variable, as this method is for RNA extraction (see MATERIALs and METHODS).

Detection of the $40 S$ rRNA precursor in the nuclear fraction of embryos at various stages. Our findings imply that the increasing amounts of 40S rRNA precursor after the late blastula stage are due to the initiation of rRNA synthesis, as based on findings in the zygotic genome. The newly synthesized 40S rRNA precursor can be detected only in the nucleus. Therefore, we investigated whether or not the 40S rRNA precursor is present in the early embryonic nuclear fraction, as described in Materials AND Methods. About $90 \%$ of the nuclei sedimented to the bottom of a tube at the neurula stage, and $50 \%$ of the nuclei sedimented at the midblastula stage, as determined by incorporation of ${ }^{3} \mathrm{H}$-thymidine into DNA (data not shown). The RNA present in the pellet was less than about $5 \%$ of the total, irrespective of the embryonic stage. Thus, the contamination of cytoplasmic fraction into the nuclear one does not occur to any significant extent.

RNAs extracted from the nuclear fraction of wild-type embryos were analyzed by Northern hybridization using ${ }^{32} \mathrm{P}$-labeled pXlrT31 as a probe. The nuclear RNAs extracted from the same number of embryos were electrophoresed. As shown in Fig. 6, the 40S rRNA precursor was not detected until the midblastula stage (Fig. 6, lane 2 ), but was clearly evident at the late blastula stage (Fig. 6, lane 3). Since the number of nuclei increases dramatically during these stages, Northern analysis with nuclear RNAs from equal numbers of nuclei at stages 8 and 9 was carried out. The same result was obtained (data not shown).

These results show that the 40S rRNA precursor present in the embryo after the 
late blastula stage is newly synthesized, thereby suggesting that the new synthesis of rRNA begins at the stage between midblastula and late blastula.

\section{DISCUSSION}

We studied the origin and change in the amount of 40S rRNA precursor in Xenopus embryogenesis by using Northern analysis to examine maternal 40S rRNA degradation and embryonic 40S rRNA synthesis. Use of mutants is an appropriate method of analyzing developmental gene expression, as established in several organisms: Drosophila, Nematode, etc. In Xenopus, evidence for the 40S rRNA precursor in embryos was obtained by investigating the wild-type embryos. In the present study, using the anucleolate mutant, we clarified that the 40S rRNA precursor in cleaving embryos is maternally inherited and remains undegraded at least until midblastula.

pXlr14D, which contains only the external transcribed spacer, has been used to detect the 40S rRNA precursor, but the low sensitivity of this probe has made it difficult to detect the 40S rRNA molecules in early embryos (3, our unpublished result). In contrast, $\mathrm{pXIrT} 31$, which contained the external transcribed spacer and a part of the 18S rRNA genes, gave a higher sensitivity (about 20-fold) for the detection of the 40S rRNA precursor. The use of the highly-sensitive probe made feasible detection of the intact molecules of 40S rRNA precursor, not detected in cleaving embryos in previous experiments.

The results of the present study are schematically illustrated in Fig. 7, which compares of the autoradiograph in Fig. 3 with the results from Figs. 5 and 6 . We found that the amount of maternal 40S rRNA precursor was constant until the midblastula stage (stage 8), decreased at the late blastula stage (stage 9), and disap-

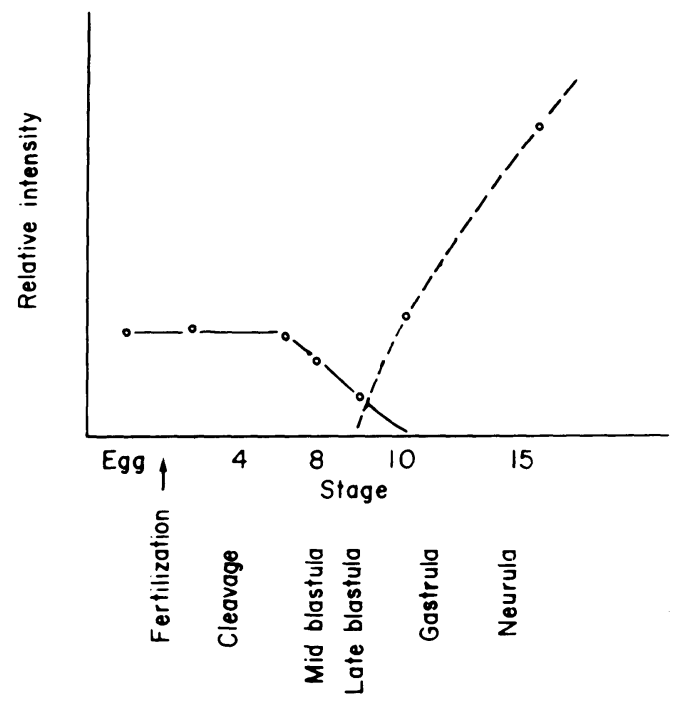

Fig. 7. Replacement of the maternal 40S rRNA precursor by newly synthesized precursor during Xenopus laevis embryogenesis. The vertical axis shows the relative intensity of the 40S rRNA precursor band when Fig. 3 was scanned by the densitometer. The change of relative intensity of maternally inherited 40S rRNA precursor (-O) and the newly synthesized one (- $(-)$ are shown in Figs. 5 and 6. 
peared at the later stages, while the newly synthesized 40S rRNA precursor increased after the late blastula stage. Thus, exchange of the newly synthesized precursor for the maternal precursor occurred during the blastula stage. A similar phenomenon has been noted for the replacement of the poly $\mathrm{A}^{+}$RNA (11) in Xenopus embryogenesis.

The question of why the 40S rRNA precursor persists during the cleavage stages even though it is an unstable RNA species has to be given attention. Gelhand and Smith (4) found that all unstable classes of nuclear RNA showed a substantial increase in stability following GVBD. Synthesis of new rRNA on embryonic rDNA overlaps the degradation of the maternal 40S rRNA (see Figs. 3 and 7). Our observations suggest the presence of a mechanism which protects the RNAs from degradation or processing in the early embryo. Mohun et al. (7) suggested that the cytoskeletal mRNA was constant before gastrulation. This mechanism may be associated with storage of the maternal RNAs. Or, extrachromosomal nucleoli may be involved in the persistence of 40S rRNA precursor, as previously discussed by Busby and Reeder (3).

Previously obtained results on rRNA initiation $(8,13)$ were based on the analyses of whole embryonic RNAs. Our analyses of nuclear RNAs lends support to the idea that the de novo rRNA synthesis is initiated at the blastula stage (Fig. 6). This seems to be the first evidence on nuclear 40S rRNA precursor which shows that rRNA genes were activated after the midblastula stage, as we proposed when the RNA labelling experiments were done (13).

Acknowledgements. We thank Dr. K. Yamana for advice and discussion, Dr. R. H. Reeder for the gift of rDNA clones, and M. Ohara for comments on the manuscript.

\section{REFERENCES}

1. Bakken, A.H., G. Morgan, B. Solner-Webb, J. Roan, S. Busby and R.H. Reeder. Mapping of transcription initiation and termination signals on Xenopus laevis ribosomal DNA. Proc. Natl. Acad. Sci. U.S.A. 79, 56-60, 1982

2. Brown, D.D. and J.B. GuRdon. Absence of ribosomal RNA synthesis in the anucleolate mutant of Xenopus laevis. Proc. Natl. Acad. Sci. U.S.A. 51, 139-146, 1964

3. Busby, S.J. and R.H. Reeder. Fate of the amplified nucleoli in Xenopus laevis embryos. Dev. Biol. 91, 458-467, 1982

4. Gelhand, R.A. and L.D. Smirh. RNA stabilization and continued RNA processing following nuclear dissolution in maturing Xenopus laevis oocytes. Dev. Biol. 99, 427-436, 1983

5. Gurdon, J.B. The control of gene expression in animal development. Clarendon Press, Oxford, 1974

6. Landesman, R. and P.R. Gross. Patterns of macromolecule synthesis during development of Xenopus laevis II. Identification of the 40S precursor to ribosomal RNA. Dev. Biol. 19, 244-260, 1969

7. Mohun, T.J., S. Brennan, N. Dathan, S. Fairman and J.B. Gurdon. Cell type specific activation of actin genes in the early amphibian embryo. Nature (London) 311, 716-721, 1984

8. NeWPORT, J. and M. KIRSCHNER. A major developmental transition in early Xenopus embryos: II Control of the onset of transcription. Cell 30, 687-696, 1982

9. Nieuwkoop, P.D. and J. FABER. Normal table of Xenopus laevis (Daudin). North Holland Publishing Co., Amsterdam, 1956

10. Rigby, P.W.J., M. Dieckmann, C. Rhodes and P. Berg. Labelling deoxyribonucleic acid to high specific activity in vitro by nicktranslation with DNA polymerase I. J. Mol. Biol. 113, 237-251, 1977

11. Sagata, N., K. Shiokawa and K. Yamana. A study on the steady state population of poly $\mathbf{A}^{+}$ 
RNA during early development of Xenopus laevis. Dev. Biol. 77, 431-448, 1980

12. Shiokawa, K. and K. Yamana. Pattern of RNA synthesis in isolated cells of Xenopus laevis embryos. Dev. Biol. 16, 368-388, 1967

13. Shiokawa, K., Y. Misumi and K. Yamana. Demonstration of rRNA synthesis in pre-gastrular embryos of Xenopus laevis. Develop. Growth and Differ. 23, 579-587, 1981

14. Southern, E.M. Detection of specific sequences among DNA fragments separated by gel electrophoresis. J. Mol. Biol. 98, 503-517, 1975

15. Tномаs, P.S. Hybridization of denatured RNA and small DNA fragments transferred to nitrocellulose. Proc. Natl. Acad. Sci. U.S.A. 77, 5201-5205, 1980

16. ThомаS, P.S. Hybridization of denatured RNA transferred or dotted to nitrocellulose paper In Methods in Enzymology, Vol. 100, Recombinant DNA part B, eds. WU, R., L. GROSSMAN and K. MOLDAVE, Academic Press, New York, pp. 255-266, 1983

17. Woodland, H.R. Change in the polysome content of developing Xenopus laevis embryos. Dev. Biol. 40, 90-101, 1974

(Received for publication, January 6, 1988) 